the liver and even then very few hepatocytes were transduced. Today, the technique has improved so much that nearly $100 \%$ of the liver can be transduced simply by injecting the vector into a mouse's tail, a particularly convenient site.

The solution, say the researchers, is to test many approaches, realizing that many will fail. "RNAi is sort of in a honeymoon period, and there will be some limitations that come out of this," says Kay, "that doesn't mean we shouldn't push the technology."

1. Lee, N.S. et. al. Expression of small interfering RNAs targeted against HIV-1 rev transcripts in human cells. Nat Biotechnol. 20, 500-505 (2002).

2. Paul, C.P., Good, P.D., Winer, I. \& Engelke, D.R. Effective expression of small interfering RNA in human cells. Nat. Biotechnol. 20, 505-508 (2002).

3. McCaffrey, A.P. et al. Inhibition of hepatitis B virus in mice by RNA interference. Nat. Biotechnol. 21, 639-644 (2003).

4. Xia, H., Mao, Q., Paulson, H.L. \& Davidson, B.L. siRNA-mediated gene silencing in vitro and in vivo. Nat. Biotechnol. 20, 1006-1010 (2002).

5. Harper, S.Q. et al. RNA interference improves motor and neuropathological abnormalities in a Huntington's disease mouse model. Proc. Natl. Acad. Sci. USA 102, 5820-5825 (2005)

6. Xia, H. et al. RNAi suppresses polyglutamine-induced neurodegeneration in a model of spinocerebellar ataxia. Nat. Med. 10, 816-820 (2004)

7. Reynolds, A. et al. Rational siRNA design for RNA interference. Nat Biotechnol. 22, 326-330 (2004).

8. Jackson, A.L. et al. Expression profiling reveals offtarget gene regulation by RNAi. Nat. Biotechnol. 21, 635-637 (2003).

\section{Golden harvests}

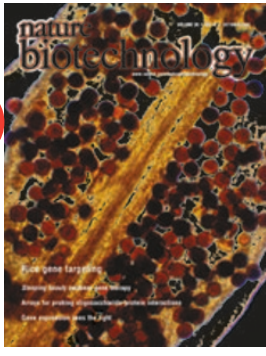

Technologists are used to trade-offs. Better sensitivity means worse specificity. Higher purity means lower yield. And for plants, greater tolerance to stress means stunted growth. Kazuo Shinozaki and Kazuko Yamaguchi-Shinozaki, a husband-wife team, and colleagues at RIKEN Plant Science Center (Yokohama, Japan), demonstrated that the last trade-off can be overcome ${ }^{1}$.

By the late 1990s, many genes that protect plants against harsh environmental conditions had been identified, but researchers balked at the prospect of transferring genes one by one into transgenic plants. Shinozaki and colleagues engineered Arabidopsis thaliana to constitutively overexpress the transcription factor DREB1A, which they had previously found to upregulate several genes in response to cold and dehydration. The transgenic plants showed remarkable increases in tolerance to freezing, water stress and salinity, but the plants were dwarf in phenotype. The researchers got around this by expressing the protective gene just when it was needed. When they used the promoter from a dehydration-induced gene to control expression of DREB1A, the resulting plants were even more stress tolerant than constitutively active transgenics and furthermore, grew to a normal size.

"The DREB1A transcription factor is a master switch of stress-inducible gene expression," explains Shinozaki. Though the research was carried out in A. thaliana, he says, "our method [for] molecular breeding of abiotic stress tolerance can be applied to real breeding of crops and trees." Shinozaki's colleagues and collaborators are currently using these new insights to probe stress responses in a wide variety of crop plants, including rice, wheat, tobacco, corn, petunia, poplar, canola, tomato, tea, and soybean. It's possible that farmers breeding crops for higher yields have, over the centuries, selected variants less likely to express protective transcription factors $^{2}$. Now, plant scientists have insights on how to tweak coordinated gene expression systems to improve stress tolerance.

Although creating more stress-tolerant crops is likely to benefit farmers everywhere, public mistrust of agricultural biotechnology remains a constraint to acceptance and expansion of the field. This is fuelled by the view that large agribusiness is the primary beneficiary of some of the current crop of transgenics, such as those displaying insectand pesticide-resistance. Efforts to produce low trans fat soybean oils or to enhance the nutritional content of products are therefore likely to be of increasing importance, but will require more complex genetic engineering than was previously required for single gene traits (e.g., in certain Bacillus thuringiensis (Bt) or herbicide-resistant crops) as well as a comprehensive analysis of plant metabolic pathways (see Box 1). In this context, the
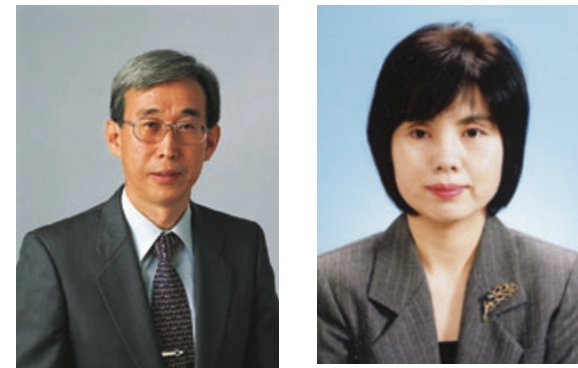

Kazuo Shinozaki and Kazuko YamaguchiShinozaki of RIKEN Plant Science Center showed that overexpression of transcription factor DREB1A confers remarkable increases in plant tolerance to freezing, water stress and salinity.

work of Rachel Drake and her colleagues at Syngenta (Basel) in enhancing $\beta$-carotene enriched rice ${ }^{3}$ (also known as 'Golden Rice') represented a significant step forward. The work continued on from a landmark study in 2000 by Ingo Potrykus and colleagues ${ }^{4}$ that aimed to produce a rice strain that could help alleviate vitamin A deficiencies, which can cause blindness among malnourished populations. The problem with the original paper was that the levels of $\beta$-carotene (a precursor to vitamin A) produced were too low to be a practical source of the nutrient. Drake and her colleagues examined the metabolic pathway in transgenic rice and found that the original daffodil gene for phytoene synthase was the limiting step in $\beta$-carotene accumulation. By systematically substituting the daffodil gene with homologs from other plants, the Syngenta team boosted pro-vitamin A levels in the rice by more than 20 -fold, which might be enough to make a practical difference in nutrition. What's more, Syngenta has donated the rights to this rice to the Golden Rice Humanitarian Board.

1. Kasuga, M., Liu, Q., Miura, S., Yamaguchi-Shinozaki, K. \& Shinozaki, K. Improving plant drought, salt, and freezing tolerance by gene transfer of a single stress-inducible transcription factor. Nat Biotechnol. 17, 287-291 (1999).

2. Smirnoff, N. \& Bryant, J.A. DREB takes the stress out of growing up. Nat. Biotechnol. 17, 229-230 (1999).

3. Paine, J.A. et al. Improving the nutritional value of Golden Rice through increased pro-vitamin A content. Nat. Biotechnol. 23, 482-487 (2005).

4. Ye, X. et al. Engineering the provitamin A (beta-carotene) biosynthetic pathway into (carotenoid-free) rice endosperm. Science 287, 303-305 (2000). 